\title{
The Value of Vision
}

\author{
Patrick Fitch
}

\section{A vision is not just a picture of what could be; it is an appeal to our better selves, a call to become something more.} —Rosabeth Moss Kanter, Harvard Business School

M ost readers of this journal are pharmacists working in hospitals, and I'm sure that each of these organizations has a Vision statement. No two Vision statements will be the same, but they all serve essentially the same purpose: to describe what an organization would like to achieve or accomplish. In other words, an organization's Vision functions as a clear guide for choosing a course of action (Business Dictionary, WebFinance Inc; www.businessdictionary.com/definition/vision-statement.html).

I hope that each of you is reasonably familiar with your employer's Vision. At the very least, good Vision statements are meant to inspire employees. In my organization, the Vision statement is one yardstick against which we are evaluated during our annual performance review.

Beyond your organization's Vision statement, how many of you have a personal Vision statement, for either your personal life or your career? If you have one, when was the last time you reviewed or updated it? When job interviewers ask the stereotypical interview question "Where do you see yourself in 5 years?" what they are really asking is "What is your Vision for your career?" Unless you have put some thought into this beforehand, the answer to that question can be less than inspiring for potential employers. In more pragmatic terms, if you don't have a plan for your career, no one else is going to create one for you.

Your career Vision needn't be a grand, all-encompassing manifesto. In many ways, simpler is better. At its heart, your Vision should define what success and excellence look like to you; it should express your view of where you want to be in the future; and it should reflect your personal values, goals, and purpose ("How to Create a Personal Mission and Vision Statement for Your Career" by Tatyana Sussex; https://www.liquidplanner.com/ blog/how-to-create-a-personal-mission- and-Vision-statementfor-your-career/).
This article isn't intended as a primer on how to create your Vision. There is no single best approach, and a multitude of helpful resources are a few clicks away on the internet. I encourage you to explore.

Once you have created your Vision, refer to it often. After all, it is meant to inspire and guide you in your career. Take time to review and update it periodically. Every 5 years is reasonable, but career and life milestones should also prompt a review.

You may be wondering about my own career Vision. In truth, it's time for me to re-evaluate and update my Vision. I'm now 10 years—or less—from retirement, and my employer is currently part of the largest restructuring of healthcare in Manitoba history. These events prompted me to review my Vision, which in turn inspired me to write this commentary.

In the meantime, take a few minutes to reflect on the Vision of the Canadian Society of Hospital Pharmacists (CSHP): "CSHP will be a thriving, progressive society, leading and inspiring excellent pharmacy practice integral to patient-centred care in hospitals and other collaborative healthcare settings."

Patrick Fitch, BSP, ACPR, is President and Internal Liaison for the Canadian Society of Hospital Pharmacists. 